\title{
Reliability and validity of the Microsoft Kinect for assessment of manual wheelchair propulsion
}

\author{
Rachel Milgrom, BS, OTD/S; ${ }^{*}$ Matthew Foreman, BSME; John Standeven, PhD; Jack R. Engsberg, PhD; Kerri \\ A. Morgan, PhD, OTR/L, ATP \\ Program in Occupational Therapy, Washington University School of Medicine, St. Louis, MO
}

\begin{abstract}
Concurrent validity and test-retest reliability of the Microsoft Kinect in quantification of manual wheelchair propulsion were examined. Data were collected from five manual wheelchair users on a roller system. Three Kinect sensors were used to assess test-retest reliability with a still pose. Three systems were used to assess concurrent validity of the Kinect to measure propulsion kinematics (joint angles, push loop characteristics): Kinect, Motion Analysis, and Dartfish ProSuite (Dartfish joint angles were limited to shoulder and elbow flexion). Intraclass correlation coefficients (ICCs) revealed good reliability (0.87-0.99) between five of the six joint angles (neck flexion, shoulder flexion, shoulder abduction, elbow flexion, wrist flexion). ICCs suggested good concurrent validity for elbow flexion between the Kinect and Dartfish and between the Kinect and Motion Analysis. Good concurrent validity was revealed for maximum height, hand-axle relationship, and maximum area (0.92-0.95) between the Kinect and Dartfish and maximum height and hand-axle relationship (0.89-0.96) between the Kinect and Motion Analysis. Analysis of variance revealed significant differences $(p<0.05)$ in maximum length between Dartfish (mean $58.76 \mathrm{~cm}$ ) and the Kinect $(40.16 \mathrm{~cm}$ ). Results pose promising research and clinical implications for propulsion assessment and overuse injury prevention with the application of current findings to future technology.
\end{abstract}

Key words: assessment, Dartfish, manual wheelchair, Microsoft Kinect, motion analysis, propulsion, reliability, upper limb, validity, video motion capture.

\section{INTRODUCTION}

Human motion analysis is utilized throughout the rehabilitation process for objective quantification and identification of baseline abilities, pathological conditions with corresponding functional effect, and improvement monitoring to determine next steps [1-3]. Human motion analysis is of particular importance in the rehabilitation of manual wheelchairs users (MWUs), considering the prevalence of pathological conditions from the overuse of the upper limbs during manual wheelchair propulsion. Manual wheelchair propulsion requires repetitive use of the upper limbs, leading to increased stress on muscles and joints, often resulting in carpal tunnel syndrome, rotator cuff tendinopathy, shoulder pain, and nerve injuries [4-8]. Seventy percent of MWUs experience an overuse injury with a reported negative effect on functional activities such as self-care and transfers [910]. Chronic overuse injuries have been attributed to improper biomechanics and poor ergonomics during

\footnotetext{
Abbreviations: ANOVA $=$ analysis of variance, ICC $=$ intraclass correlation coefficient, MWU = manual wheelchair user, SDK = software development kit.

*Address all correspondence to Rachel A. Milgrom, BS, OTD, OTR/L; 2228 S 11th St, St Louis, MO 63104; 614403-4882. Email: milgromrachel@gmail.com

http://dx.doi.org/10.1682/JRRD.2015.10.0198
} 
wheelchair propulsion [9,11-12]. Unfortunately, current options for the objective assessment of manual wheelchair propulsion are limited because of monetary, temporal, and portability restrictions. Such restrictions are often dependent upon whether the system yields two- or threedimensional data.

\section{Three-Dimensional Analysis}

Three-dimensional human motion analysis systems are divided into non-visual-based and, more frequently, visual-based systems. In visual-based systems, optical sensors (i.e., cameras) are employed to improve accuracy in measuring movement. Visual-based systems are then further categorized into marker-based or marker-free. Visual-marker-based systems utilize optical sensors in combination with "markers" adhered to the human body at anatomical bony landmarks corresponding to joints of interest. Visual-marker-based systems are frequently used in research and are considered the "gold standard" in human motion analysis because of a typical accuracy of $0.5 \mathrm{~mm}$ with which the optical sensors are able to locate and quantify each marker's position [13]. The majority of research on manual wheelchair propulsion has been conducted using the gold standard [14-18]. However, several limitations to the use of visual-markerbased systems exist. Cluttered environments, varied lighting, overlapping body parts during rotation, unreliable identification of bony landmarks, marker movement, and potential marker detachment may all affect the accuracy of marker detection and location [13,19]. Perhaps most notably, visual-marker-based systems are also very expensive, costing upwards of $\$ 150,000,{ }^{*}$ and are difficult to use [20], requiring extensive time and expertise for both setup and implementation, limiting their use in clinical and community settings.

A more cost-effective alternative is a visual-markerfree system utilizing optical sensors (cameras) alone. Cameras with high enough resolution have the ability to achieve adequate accuracy in detecting movement [13]. However, system limitations include the need for sufficient camera bandwidth and complex postprocessing methods to transform two-dimensional video data into three dimensions, reduce errors, and minimize latency, which all potentially decrease data accuracy [21].

\footnotetext{
* P. Hagerman (Director of Sales, Motion Analysis Corporation; Santa Rosa, CA). Personal communication with: J. R. Engsberg. 2008 Jun 2.
}

\section{Two-Dimensional Analysis}

Two-dimensional options in human motion analysis include manual digitization of video recordings and the use of software programs to automatically track passive markers placed on the performer by the evaluator (e.g., APAS, ImageJ, Hu-m-an, Qualisys). The software package Dartfish ProSuite 5.5 (Fribourg, Switzerland) suggests potential for clinical analysis of human motion as an alternative to its three-dimensional, gold-standard counterparts with increased portability [20,22-23]. The system has the basic requirements of a standard video and computer and has already been used in the analysis of wheelchair sport propulsion biomechanics [24-25]. While Dartfish utilizes only a video camera to obtain twodimensional data of human motion, the software program required for analysis still presents a costly barrier at an average price of $\$ 3,800 .^{\dagger}$ The program allows for the visual location of anatomical landmarks and manual marker assignment for automatic tracking during a replay of the previously recorded video file. Additional measurements, such as joint angles, must also be manually measured and tracked with the software-provided "tools," increasing analysis time and risk of human error.

\section{Microsoft Kinect}

The Microsoft Kinect (Kinect for 360, Microsoft; Redmond, Washington) may serve as a potential human motion analysis alternative, maintaining the accuracy of three-dimensional systems as well as the cost-effectiveness and portability of two-dimensional analysis. The Kinect is a visual-based tracking system that uses a single redgreen-blue camera and depth sensor to provide a full body, three-dimensional motion capture based on anatomical landmarks and joint positions without markers or handheld controllers [26]. While the Kinect was originally designed for gaming, it is currently being used as a rehabilitation tool for intervention in stroke [26], cerebral palsy [27], cognitive dysfunction [28], and fall prevention [29]. The Kinect is also being used for the assessment of postural control [30], gait [31], balance [3], static foot posture [32], body sway [33], and common motor movements [34], all with promising comparisons to three-dimensional gold standard systems. The use of the Kinect as a rehabilitation tool outside of the clinic and in

\footnotetext{
${ }^{\dagger}$ C. Wilmot (Dartfish Region Manager, Dartfish; Fribourg, Switzerland). Personal communication with: R. Milgrom. 2015 May 8.
} 
the home [35-37] suggests the sensor's portability and supports the recommendation of previous research to incorporate assessment throughout the continuum of care [38].

While the use of the Microsoft Kinect as an assessment tool is becoming more prevalent, a gap in the literature still exists. There is currently no research pertaining to the use of the Kinect with the MWU population to quantify wheelchair propulsion motion. The current study proposes the Kinect's potential to provide an additional rehabilitation assessment tool comparable to the currently available options. Therefore, the objectives of the current study are to determine interrater and testretest reliability of the Kinect sensor in addition to its concurrent validity by comparing outcomes to commonly used three-dimensional and two-dimensional systems.

\section{METHODS}

This study utilized a group design in which participants each completed a single assessment session. The protocol for data collection and participant interaction was approved by the Washington University Human Research Protection Office.

\section{Participants}

Five adults (four men, one woman) who use a manual wheelchair were recruited for this pilot investigation. Four participants had a spinal cord injury (three with tetraplegia, one with paraplegia), and were right-hand dominant. The fifth participant had a disability from birth resulting in disproportionate limbs and identified as ambidextrous. All participants had an ultra-lightweight wheelchair frame, two with 24 in. rear wheels and three with 25 in. rear wheels. Recruitment resources included a local independent living center and a wheelchair sports team. Participants were screened upon response to a recruitment flyer to ensure that they met the following inclusion criteria: possessed the ability to self-propel a manual wheelchair, used a wheelchair for at least 75 percent of daily activities, were at least 18 yr of age, understood spoken English, and were able to provide informed consent. Informed written consent was obtained from all participants prior to participation in this study.

\section{Assessment Tools}

\section{Microsoft Kinect}

The Microsoft Kinect sensor is a portable, inexpensive motion-sensing device that can be used in conjunction with a personal computer to monitor the threedimensional position and orientation of a person's body segments without the use of reflective markers or wearable devices [31]. During data collection, one laptop computer and one Kinect sensor were placed in front and to the right of the participant on top of a wooden stand $1.5 \mathrm{~m}$ high, angled toward the center of the participant's right wheel axle to measure the movement of the head; neck; torso; and right shoulder, elbow, wrist, and hand at a sampling rate of approximately $30 \mathrm{~Hz}$. Two other Kinect sensors were interchanged with the original sensor in between each trial for a total of three sensors, one for each trial. All sensors utilized "seated" mode, available in the Microsoft Kinect software development kit (SDK) (version 1.8). This allowed only landmarks of upper-body structures to be tracked by the Kinect sensors.

Interference between infrared Kinect sensor signals prevented the use of all three sensors simultaneously. While theoretically sensor properties should be the same, interchanging sensors between trials allowed for testing of this theory with each sensor serving as its own "rater" to establish interrater reliability. The Kinect sensor has never been tested in this application. Therefore, interrater reliability must first be established before meaningful comparisons may be made in regard to validity outcomes. Consistent placement of the Kinect sensor aimed to reduce human variability to more closely capture the sensor's interrater reliability potential.

\section{Dartfish}

Videos taken during the assessment were analyzed using Dartfish ProSuite 5.5 Video Software Solutions. Dartfish tracks kinematic movement in two-dimensional videos. One video camera (Canon FS200; Tokyo, Japan) was used during data collection; it was positioned at the level of the right wheel axle, with the frame capturing the area from the bottom of the wheel to the participant's right, dominant shoulder.

\section{Motion Analysis}

Motion Analysis (HiRes Motion Analysis Corporation System, Motion Analysis Corp; Santa Rosa, California) is an expensive visual-marker-based motion tracking system 
with a sampling rate set at $60 \mathrm{~Hz}$. Eight infrared cameras mounted from the ceiling encircled the participant to locate and track the movement of the 41 reflective markers placed on the participant's head, arms, trunk, knees, and wheelchair. The markers were adhered to anatomical bony landmarks and structural components of the participant's wheelchair.

\section{Assessment Procedures}

\section{Motion Analysis Calibration}

The eight cameras were calibrated before collecting motion data. All cameras were aimed to sufficiently capture the space in which the participant was positioned. Additionally, the capture volume was checked for reflective materials that may be mistaken as a marker. This was completed at the time of calibration and repeated following participant preparation to verify that the system was viewing exactly 41 markers.

\section{Participant Preparation}

Following the participant's arrival, the principal investigator obtained informed consent. Forty-one reflective markers were then adhered to the participant's head, trunk, shoulders, elbows, wrists, hands, knees, and wheelchair. Participants had the choice to be shirtless or wear a tank top/sports bra to eliminate clothing interference and allow for more direct adherence to the skin for the majority of markers. The participant was safely spotted onto the roller system with straps to secure the wheelchair. Once in position, the principal investigator verified that the appropriate body segments were being captured on the two digital video cameras: one paired with the Motion Analysis system and one used for biofeedback during static trials.

\section{Kinect Calibration}

At the beginning of the assessment session, the sensor was placed in an initial starting position in front of and to the right of the participant and angled in slightly. This starting position was established by previous trials as able to collect relatively consistent, accurate data and previous work suggesting the inability of the Kinect sensor to obtain accurate data when placed directly in front of the participant [34]. In the event that the same accurate data were unable to be obtained during calibration, the sensor and platform were repositioned and moved slightly to the right and farther from the participant while maintaining the same angle before proceeding further.
This would compensate for the discrepancy in height between participants. Last, the participant was asked to place the center of his or her right palm over the right wheel hub while the Kinect recorded its position for $30 \mathrm{~s}$ for later use as a frame of reference in determination of the hand-axle relationship.

\section{Static Position Frame of Reference}

A 32 in. television monitor was placed on a table directly in front of the participant and outside the capture volume. A large mirror stood on the ground next to the television, angled toward the participant. A standard video camera (Canon FS200) was connected to the television and captured the participant through the mirror. The participants were instructed to look at themselves in the television screen. Aiming the camera through the mirror allowed for movement of the participant's right upper limb to be seen as movement on the right side of the screen. This allowed for increased ease of position replication-creation as opposed to a right-left "mirrored" effect when the camera was pointed directly at the participant. The participant was then asked to place his or her arms in a " $U$ " shape with palms facing forward and to hold the position (Figure 1). An outline of this position was drawn on the TV screen with a dry erase marker to assist participants in maintaining the same position across several trials because of our inability to use three sensors simultaneously. This particular static position was selected based on previous research [39] and need for a position with limited joint rotation due to the two-dimensional nature of the video feedback for participant repositioning.

\section{Static Trials}

Static trials were completed to establish reliability outcomes. The purpose of the first series of static trials was to establish interrater reliability, with three Kinect sensors each serving as an individual "rater." The second series was to establish test-retest reliability, with comparisons made between a single sensor across the first and second series of trials. Static trials required the participant to maintain a still or "static" position. At the beginning of each static trial, the Kinect sensor and corresponding laptop, along with the Motion Analysis system and connected video camera for Dartfish analysis, began recording simultaneously with a verbal countdown. Participants were cued and given 10 to $12 \mathrm{~s}$ to raise their arms and reposition themselves with the outline drawn on the television screen; they were then told to hold this position for $30 \mathrm{~s}$. After 


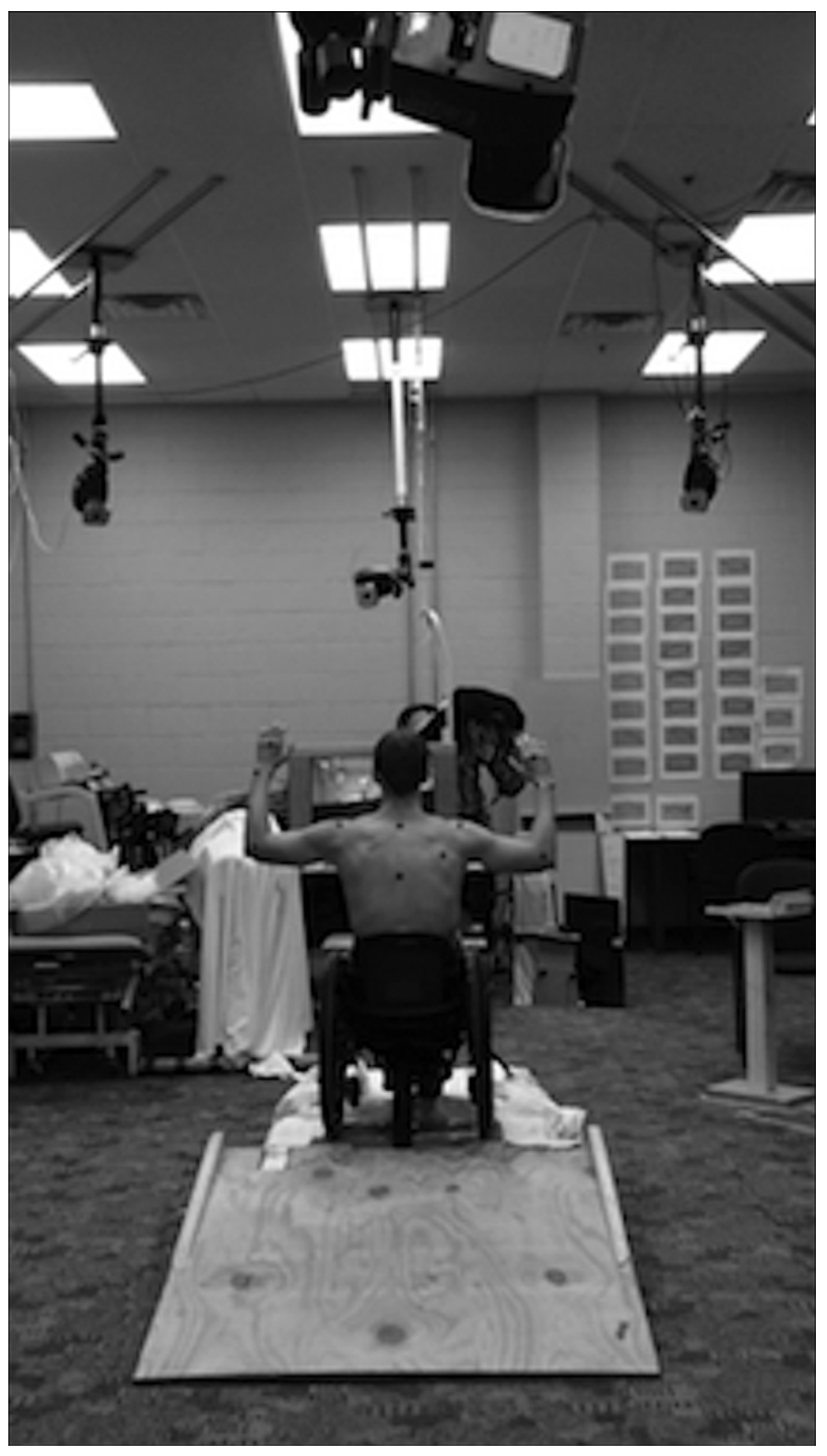

Figure 1.

Static position frame of reference.

each $42 \mathrm{~s}$ trial, the first Kinect sensor, labeled "Kinect 1," was replaced with the second sensor (Kinect 2). This same process was repeated for the third trial with Kinect 3 . After the first three trials, the laptop was shut down and the sensor was disconnected and removed from the wooden structure and set aside for 5 min while the participant remained in place. Following reassembly, an identical set of three trials with Kinect 1, 2, and 3 was completed for a total of six 42 s static trials.

\section{Dynamic Trials}

Dynamic trials were completed to establish validity outcomes with all three systems recording simultaneously, allowing for comparisons of Kinect outcomes to those obtained by the Dartfish and Motion Analysis systems at the same time points. Comparisons between the Kinect and Dartfish system were made only in relationship to the Motion Analysis system because Dartfish has not been tested for concurrent validity against the gold standard in the application of manual wheelchair propulsion assessment.

All dynamic trials required participants to perform a functional or "dynamic" movement, which was defined as propelling their wheelchairs on the stationary roller system for the same $42 \mathrm{~s}$ period, including an initial 10 to $12 \mathrm{~s}$ to gain a comfortable, consistent speed. This was repeated three times for the interchange of the three Kinect sensors. Variability in propulsion speeds across trials was not explicitly controlled for since dependent variables were only compared within trials at identical time points across systems. The same may be said for the participant's wheelchair setup. However, wheelchair setup was consistent across all trials because the participants remained in their wheelchairs and secured on the roller system for the entirety of the assessment.

An identical series of three dynamic trials was performed immediately following a short 5 min intermission in which Kinect sensors were disconnected from their power source and their driver computer powered down, but with all equipment remaining in place.

\section{Data Processing}

Data were processed for only the right, dominant upper limb for all participants, with joint angle and push loop outcome measures dependent upon trial type and system. Synchronization of data between the Kinect, Motion Analysis, and Dartfish system was aligned based on global time stamps obtained from each frame of data from each device.

\section{Static Data: Microsoft Kinect}

Depth data from the Kinect sensor were automatically converted into skeletal data using the skeleton tracking algorithm built into the SDK. Each skeletal frame was streamed into a custom written program (C\#) and saved in an output file for postprocessing. Three-dimensional skeletal data from the Kinect sensor were postprocessed using MATLAB (MathWorks; Natick, Massachusetts) to filter 
data using a second-order Butterworth filter with a cutoff frequency of $1 \mathrm{~Hz}$ to obtain outcome measures. Outcome measures included joint angles of the neck (flexion), trunk (flexion), shoulder (flexion, abduction), elbow (flexion), and wrist (flexion). Joint angle calculations were based on standard biomechanical analyses [40]. However, methods were adjusted because orientation data from the Kinect was not collected and the anatomical landmarks inherent to the Kinect do not provide enough markers on each body segment to create a full local coordinate system. Therefore, methods were primarily based on common clinical definitions of standard planar angles [41]. For example, shoulder flexion was calculated as the angle between the humerus vector (shoulder to elbow) and the torso vector (neck to shoulder midpoint) in the sagittal plane. For identifying torso movement, a virtual landmark located at the midpoint of the shoulders was created during postprocessing since "seated mode" does not include a torso landmark. A vector between this shoulder midpoint and the neck landmark measured by the Kinect was also created and compared to the global vertical axis in multiple planes to calculate comprehensive trunk angles.

Angles were initially obtained for all time points across the middle five consecutive seconds of each $42 \mathrm{~s}$ trial and then averaged. The use of the middle five seconds was chosen to account for the difference in data during the initial alignment with the static position frame of reference as well as fatigue at the end of the trial.

\section{Dynamic Data}

Microsoft Kinect. Depth data from the Kinect sensor were automatically converted into skeletal data using the skeleton tracking algorithm built into the SDK. Each skeletal frame was streamed into a custom-written program $(\mathrm{C \#})$ and saved in an output file for postprocessing. Threedimensional skeletal data from the Kinect sensor were postprocessed with MATLAB and the Butterworth filter technique utilized for static data. Outcome measures for all three systems included joint angles as measured in static trials, along with push loop characteristics. Because of the lack of wheelchair markers and oblique position of the Kinect sensor relative to the chair, push loop characteristics had to be calculated in three dimensions (xyz). Push loop characteristics were defined as follows: push length (distance traversed by the trajectory of the hand during a full push loop), maximum loop length (distance between the maximum and minimum lateral positions of the hand from the wheel hub during the push loop), maximum loop height (distance between the maximum [positive, above] and minimum [negative, below] vertical positions of the hand from the wheel hub during the push loop), total loop area (3.14 $\times 1 / 2$ max length $\times 1 / 2$ max height), and hand-axle relationship (minimum straightline distance between the hand and the wheel hub during the push loop) [42]. To define each push loop, an algorithm was developed to determine the frame at which the hand landmark was both at a maximum vertical distance in the global $x y$ plane and at a minimum lateral distance from the wheel hub. The position of the wheel hub was determined from an initial static trial during which the participant was asked to hold his or her hand over the wheel hub for $30 \mathrm{~s}$. A $15 \mathrm{~s}$ average from this trial of the $x y z$ hand position was used as an estimate of the wheel hub position. Full push cycles were determined as all frames between each "top center" frame when the third metacarpal is directly above the axle of the wheelchair.

Data were processed from the five consecutive middle pushes during each $42 \mathrm{~s}$ trial, resulting in five individual values for each joint angle outcome and push loop characteristic. Joint angle outcomes were then obtained from a single Kinect sensor (Kinect 1), since sufficient interrater reliability had been established in static trials, from the first trial of each dynamic series at the top center time point of all fives push loops for continued comparison. Push loop characteristics were analyzed using the same trials and five pushes from the same single sensor.

Dartfish. Videos were uploaded to Dartfish ProSuite 5.5; the five push loops analyzed were determined based on the corresponding Motion Analysis time stamps. Because of the difference in system frame rates, the top center of the first push was visually established using Dartfish when the third metacarpal marker was directly above the axle. If off significantly, the difference was calculated between Dartfish-provided time stamps and the Motion Analysis top center time stamp. The difference was applied to all five pushes in the trial. In the event that the Motion Analysis time stamp was between two Dartfish frames, the time stamp one frame ahead was chosen. Data were then processed using Dartfish's angle tracking and distance tool to obtain outcome measures based on established methods.

Outcomes included two-dimensional joint angles of the shoulder flexion/extension (lateral epicondyle-acromionwheel axle hub) and elbow flexion/extension (acromionlateral epicondyle-third metacarpal), along with push 
loop characteristics (push length, maximum loop length, maximum loop height, total loop area, and hand-axle relationship) as defined by the Kinect system. The angletracking tool established joint angle outcomes as well as push angle to be converted into push length ([push angle $\times$ $3.14 / 180] \times$ wheel radius). Push angle was defined as the beginning of the push when the third metacarpal first made contact with the push rim until the hand left the push rim; the angle was measured as the relation of the two points to the wheel axle. The distance tool established the same push loop characteristics as measured by the Kinect and Motion Analysis systems during dynamic trials.

Motion Analysis. Cortex Motion Analysis Software performed initial postprocessing. Data were then visually reviewed frame by frame to verify accurate tracking of all 41 reflective markers. Three-dimensional positioning of each marker was then exported in an output file for further processing via customized Microsoft Excel formulas to obtain push loop characteristics, as previously defined, in the sagittal (xy) plane based on established methods [43-45]. To establish the position of the hand relative to the wheel hub, the vector between the two representative markers was calculated. Each push loop was then defined by the "top center" frame at which the third metacarpal marker was directly above the wheel hub and at a minimum lateral distance from the wheel hub. Full push cycles were determined as all frames between each "top center" frame. The previously referenced MATLAB output file was also utilized for Motion Analysis data, but used the default Butterworth filter (fourth order, $6 \mathrm{~Hz}$ ) to smooth and edit for length to obtain joint angle outcomes. Standard biomechanical analyses [40] were utilized since the Motion Analysis system allowed for more direct creation of local coordinate systems for body segments and calculation of three-dimensional rotational joint kinematics. Methods were supplemented with similar clinical definitions [41] used to calculate standard planar angles and adjusted accordingly. For example, shoulder flexion was calculated as the angle between the humerus vector (acromion to epicondyle midpoint) and the torso vector (vertical axis in local coordinate system) in the sagittal plane.

A mixed methods approach was used to address outlying dynamic data from trial one in the first series of dynamic trials across systems for all variables based upon visual data inspection and descriptive statistics. Each frame from the Microsoft Kinect SDK comes with a tracking state descriptor labeled "tracked," "inferred," or "not tracked." The inferred frames often contained kinematic data that was several standard deviations away from the surrounding frames of "tracked" data. To remedy this, a moving average filter was used to impute the mean of the $x y z$ data with the mean of the surrounding tracked frames for each inferred and not tracked frame. A wrap-around technique was used to pad values at the beginning of the data set with values from the end and vice versa, facilitating a complete moving window for all frames [46].

\section{Data Analysis}

All data were imported and analyzed using SPSS version 21 (IBM; Armonk, New York). Descriptive data, including mean and standard deviations, were calculated for each trial.

\section{Static Data}

Paired $t$-tests were performed to examine interrater reliability and differences between joint angle outcome data across three Kinect sensors during Static 1 trials. Intraclass correlation coefficients (ICCs) Model 3,k were performed to examine the interrater aspect of analysis by determining how closely related joint angle outcomes were across the different Kinect sensors during Static 1 trials. Paired $t$-tests were also performed to examine testretest reliability and differences between joint angle outcome data across Static 1 and Static 2 trials within a single Kinect sensor.

\section{Dynamic Data}

Repeated-measures analyses of variance (ANOVAs) were performed with a post hoc Bonferonni analysis to examine differences in joint angle and push loop characteristic data across all three systems. ANOVAs were also used to examine whether the Kinect power down between trial 1 and trial 2 affected differences in all outcome variables between only Kinect 1 and the Motion Analysis systems. Dartfish was not included in this last ANOVA because the dynamic movements were not regulated across trials and the system has not yet been established as a reliable assessment tool for manual wheelchair propulsion, potentially skewing results with inconsistent data.

Mauchly's Test of Sphericity and Shapiro-Wilk Normality Test were used to validate ANOVAs for all outcome variables. In the event the assumption of sphericity was violated, indicated by a significant $p$-value $(p<0.05)$, the Greenhouse-Geisser $p$-value was reported. Variables 
violating the Shapiro-Wilk Normality Test for all three devices were considered to have violated the assumption of normality.

$\operatorname{ICC}(3, \mathrm{k})$ was used to examine concurrent validity by determining how closely related joint angle and push loop characteristic data were across the three systems. ICC values were interpreted based on clinical measure guidelines from Portney and Watkins ( 0.80 and above is good, and 0.50 to 0.79 is poor to moderate) [47].

\section{RESULTS}

\section{Static Data: Joint Angles}

Paired $t$-tests revealed no statistically significant joint angle differences across the three Kinect sensors. ICCs revealed good reliability (ranging from 0.87 to 0.99) between joint angle data for neck flexion, shoulder flexion, shoulder abduction, elbow flexion, and wrist flexion (Table 1). For comparisons made within sensors across trials, paired $t$-tests revealed no significant differences in any joint angle outcomes for Kinect 1 and Kinect 2. Significant differences were revealed for Kinect 3 in the measurement of shoulder abduction ( $p<0.05$; Table 2). ICCs revealed good reliability (ranging from 0.87 to 0.99) between shoulder flexion, shoulder abduction, elbow flexion, and wrist flexion for Kinect 1 and Kinect 2. For Kinect 3, ICCs revealed good reliability (ranging from 0.87 to 0.98 ) between neck flexion, trunk flexion, shoulder flexion, and shoulder abduction (Table 3).

\section{Dynamic Data: Joint Angles and Push Loop Characteristics}

ANOVA revealed no significant differences in joint angle outcome data across the three systems (Table 4). No significant differences were observed for the push loop characteristics of maximum height and hand-axle relationship (Table 5). ANOVA also revealed no significant system differences between the Kinect and Motion Analysis across trials for joint angles and push loop characteristics. ANOVA did reveal significant differences in

Table 1.

Descriptive data and interrater reliability of Kinect sensors for joint angle data.

\begin{tabular}{lcccc}
\hline \multicolumn{1}{c}{ Movement } & Kinect 1, Mean \pm SD & Kinect 2, Mean \pm SD & Kinect 3, Mean \pm SD & ICC (95\% CI) \\
\hline Neck Flexion & $13.17 \pm 5.36$ & $14.24 \pm 7.25$ & $12.30 \pm 4.31$ & $0.87(0.52-0.99)^{*}$ \\
Trunk Flexion & $22.78 \pm 5.36$ & $26.18 \pm 5.59$ & $25.44 \pm 4.64$ & $0.69(0.14-0.96)$ \\
Shoulder Flexion & $96.87 \pm 24.99$ & $93.13 \pm 19.75$ & $90.47 \pm 23.53$ & $0.97(0.84-1.00)^{*}$ \\
Shoulder Abduction & $96.53 \pm 15.73$ & $95.45 \pm 11.87$ & $93.29 \pm 10.92$ & $0.94(0.72-0.99)^{*}$ \\
Elbow Flexion & $115.43 \pm 23.71$ & $118.82 \pm 20.15$ & $120.43 \pm 19.02$ & $0.96(0.83-1.00)^{*}$ \\
Wrist Flexion & $48.18 \pm 59.12$ & $49.86 \pm 66.70$ & $53.78 \pm 66.21$ & $0.99(0.95-1.00)^{*}$ \\
\hline
\end{tabular}

Note: Means and SDs are reported in degrees. The data were taken from Trial 1 for each sensor.

${ }^{*} p<0.05$.

$\mathrm{CI}=$ confidence interval, ICC $=$ intraclass correlation coefficient, $\mathrm{SD}=$ standard deviation.

Table 2.

Descriptive data for Kinect sensors by trial for joint angle data, given as mean \pm standard deviation.

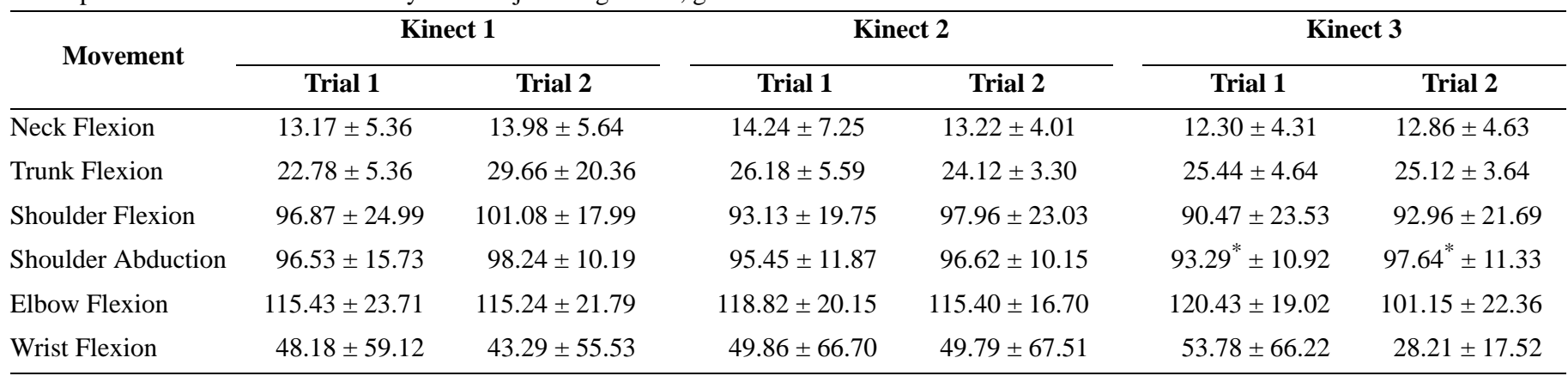


Table 2.

Descriptive data for Kinect sensors by trial for joint angle data, given as mean \pm standard deviation.

${ }^{*}$ Paired $t$-test $p<0.05$.

Table 3.

Test-retest reliability of Kinect sensors across trials for joint angle data. Intraclass correlation coefficient (95\% confidence interval).

\begin{tabular}{lllr}
\hline \multicolumn{1}{c}{ Movement } & \multicolumn{1}{c}{ Kinect $\mathbf{1}$} & Kinect 2 & Kinect 3 \\
\hline Neck Flexion & $0.77(-0.10$ to 0.97$)$ & $0.61(-0.40$ to 0.95$)$ & $0.97(0.77$ to 1.00$)$ \\
Trunk Flexion & $0.25(-0.70$ to 0.88$)$ & $0.29(-0.68$ to 0.89$)$ & $0.87(0.21$ to 0.99$)$ \\
Shoulder Flexion & $0.91(0.40$ to 0.99$)$ & $0.96(0.67$ to 1.00$)$ & $0.98(0.80$ to 1.00$)$ \\
Shoulder Abduction & $0.87(0.20$ to 0.99$)$ & $0.95(0.58$ to 0.99$)$ & $0.96(0.65$ to 1.00$)$ \\
Elbow Flexion & $0.99(0.88$ to 1.00$)$ & $0.95(0.61$ to 1.00$)$ & $-0.77(-0.97$ to 0.12$)$ \\
Wrist Flexion & $0.99(0.92$ to 1.00$)$ & $1.00(0.98$ to 1.00$)$ & $0.48(-0.54$ to 0.93$)$ \\
\hline \hline
\end{tabular}

Table 4.

Analysis of variance results (mean \pm standard deviation) for joint angle data by system.

\begin{tabular}{lccc}
\hline \multicolumn{1}{c}{ Movement } & Dartfish & Microsoft Kinect & Motion Analysis \\
\hline Neck Flexion & - & $22.56 \pm 13.42$ & $12.03 \pm 4.98$ \\
Trunk Flexion & - & $29.14 \pm 31.37$ & $35.18 \pm 5.23$ \\
Shoulder Flexion & $47.75 \pm 17.30^{*}$ & $47.32 \pm 32.92^{*}$ & $41.06 \pm 70.90^{*}$ \\
Shoulder Abduction & - & $39.65 \pm 35.95$ & $65.21 \pm 15.27$ \\
Elbow Flexion & $93.31 \pm 19.16$ & $107.85 \pm 37.13$ & $88.82 \pm 11.89$ \\
Wrist Flexion & - & $39.29 \pm 43.13$ & $36.13 \pm 5.28$ \\
*Volates Shapiro-Wilk Normality Test. & & &
\end{tabular}

Table 5.

Analysis of variance results (mean \pm standard deviation) for push loop characteristic data by system.

\begin{tabular}{lccr}
\hline \multicolumn{1}{c}{ Measure } & Dartfish & Kinect & Motion Analysis \\
\hline Maximum Length $(\mathrm{cm})^{* \dagger}$ & $58.76 \pm 7.73$ & $40.16 \pm 11.57$ & $52.48 \pm 5.44$ \\
Maximum Height $(\mathrm{cm})$ & $22.82 \pm 8.50$ & $24.55 \pm 7.42$ & $19.31 \pm 7.34$ \\
Push Length $(\mathrm{cm}){ }^{*}$ & $61.43 \pm 14.08$ & $49.70 \pm 12.30$ & $60.53 \pm 7.92$ \\
Hand-Axle Relationship $(\mathrm{cm})$ & $7.56 \pm 9.28$ & $8.51 \pm 4.60$ & $9.22 \pm 7.60$ \\
Maximum Area $\left(\mathrm{cm}^{2}\right)^{* \ddagger}$ & $1,077.15 \pm 457.44$ & $793.40 \pm 307.19$ & $679.00 \pm 283.71$ \\
${ }^{*} p<0.05$ & & \\
${ }^{\dagger}$ Bonferonni significant between Dartfish and Kinect, Dartfish and Motion Analysis. & \\
${ }^{\ddagger}$ Bonferonni significant between Dartfish and Motion Analysis. & \\
\hline \hline
\end{tabular}

push length among the three systems $(p<0.05)$. However, Bonferonni post hoc analysis, used for reduction of type I errors among multiple pairwise tests within a single data set, revealed no significant differences in push length between any particular systems. Significant differences in maximum length and maximum area were observed between systems ( $p<0.01$ ); Bonferroni post hoc analysis revealed significant differences in maximum length between Dartfish and Kinect and Dartfish and Motion Analysis, as well as significant differences in maximum area between Dartfish and Motion Analysis.
Following Mauchly's Test of Sphericity, the assumption of sphericity was violated only for maximum height among push loop outcomes, in which the GreenhouseGeisser $p$-value was not statistically significant. For joint angle outcomes, assumption of sphericity was not violated for elbow flexion or shoulder flexion. GreenhouseGeisser $p$-values were evaluated for the remaining joint angle outcomes (neck flexion, trunk flexion, shoulder abduction, and wrist flexion) since a sphericity $p$-value could not be calculated because of lack of Dartfish data for these comparisons. No Greenhouse-Geisser $p$-value 
for any outcome variable achieved statistical significance. According to the Shapiro-Wilk Normality Test, the only outcome variable to violate the assumption of normality across all three devices was shoulder flexion.

ICCs suggested good concurrent validity for the measurement of elbow flexion between the Kinect and Dartfish, as well as between the Kinect and Motion Analysis (Table 6). Good concurrent validity was also revealed for measurements of maximum height, hand-axle relationship, and maximum area (ranging from 0.92 to 0.95 ) between the Kinect and Dartfish and measurements of maximum height and hand-axle relationship (ranging from 0.89 to 0.96) between the Kinect and Motion Analysis (Table 7).

\section{DISCUSSION}

The Kinect is currently used for gaming, intervention, and assessment. This pilot study proposes a novel application of the Kinect as an assessment tool for manual wheelchair propulsion, requiring establishment of both reliability and validity outcomes.

\section{Reliability}

Results suggest that the Kinect has sufficient interrater reliability for angles measured at the neck, shoulder, elbow, and wrist joints. The reliability of these joint angles across all three tested sensors is of particular significance because

Table 6.

Concurrent validity of joint angle data. Intraclass correlation coefficient (95\% confidence interval).

\begin{tabular}{lcc}
\hline \multicolumn{1}{c}{ Movement } & Dartfish and Kinect & $\begin{array}{c}\text { Kinect and Motion } \\
\text { Analysis }\end{array}$ \\
\hline Neck Flexion & - & $-0.65(-14.81$ to 0.83$)$ \\
Trunk Flexion & - & $-0.08(-9.41$ to 0.89$)$ \\
Shoulder Flexion & $0.47(-4.07$ to 0.95$)$ & $-0.18(-10.37$ to 0.88$)$ \\
Shoulder Abduction & - & $-5.52(-61.63$ to 0.32$)$ \\
Elbow Flexion & $0.94(0.40$ to 0.99$)$ & $0.86(-0.32$ to 0.99$)$ \\
Wrist Flexion & - & $-1.12(-19.33$ to 0.78$)$ \\
\hline \hline
\end{tabular}

Table 7.

Concurrent validity of push loop characteristic data. Intraclass correlation coefficient (95\% confidence interval).

\begin{tabular}{lll}
\hline \multicolumn{1}{c}{ Measure } & Dartfish and Kinect & $\begin{array}{c}\text { Kinect and Motion } \\
\text { Analysis }\end{array}$ \\
\hline Maximum Length & $0.68(-2.08$ to 0.97$)$ & $0.41(-4.72$ to 0.94$)$ \\
Maximum Height & $0.94(0.39$ to 0.99$)$ & $0.96(0.63$ to 1.00$)$ \\
Push Length & $0.80(-0.90$ to 0.98$)$ & $0.70(-1.85$ to 0.97$)$
\end{tabular}

Table 7.

Concurrent validity of push loop characteristic data. Intraclass correlation coefficient (95\% confidence interval).

\begin{tabular}{llc} 
Hand-Axle Relationship & 0.92 (0.19 to 0.99$)$ & $0.89(-0.11$ to 0.99$)$ \\
Maximum Area & $0.95(0.55$ to 1$)$ & $-1.06(-18.75$ to 0.79$)$ \\
\hline \hline
\end{tabular}

the shoulder, elbow, and wrist joints are where the vast majority of overuse injuries attributed to wheelchair propulsion occur [4-8]. The Kinect sensors do, however, fail to reveal significant reliability among measurements of trunk flexion. For this study, the Kinect only directly captured hands, wrists, elbows, shoulders, neck, and head landmarks. The absence of an inherent trunk landmark or vector required postprocessing creation of a new point, as mentioned in the "Methods" section. Measurement of trunk flexion is important for quantification of wheelchair propulsion biomechanics, and therefore these results may be of potential clinical significance. For example, results suggest that facilities may be unable to use multiple Kinects interchangeably since calculation of this movement is limited by the scope of the technology-using off-the-shelf Kinect sensors and the readily accessible Microsoft Kinect SDK with "seated mode" does not supply an inherent, reliable trunk vector. Facilities may instead designate a single sensor for propulsion assessment after test-retest reliability has been established for said Kinect among all variables of interest. This should be noted as a limitation to the technology and prioritized for future hardware adaptation and software development considering the research that suggests the relationship between excessive trunk motion and wheelchair propulsion outcomes such as fatigue [48].

Results from the current study's test-retest reliability efforts suggest that the same Kinect sensor is not always able to obtain the same joint angle outcomes across repeated trials. One of the tested sensors (Kinect 3) was unable to obtain consistent results for elbow and wrist flexion. However, this same sensor was the only one to obtain consistent results for neck and trunk flexion. While these results do not necessarily suggest that the Kinect does not possess test-retest reliability, they may again speak more to clinical significance with regard to assessment setup. Between the two time points used to establish test-retest reliability, the equipment setup for only the Kinect system was disassembled, set aside, and reassembled. This sequence represented a clinical scenario in which an area is designated for a propulsion assessment for a limited amount of time, removed at the end of the day, and then set back up in the event of reas- 
sessment. In this case, the inability of the same Kinect to obtain similar joint angle outcomes may, in fact, reflect the inability to replicate the identical setup in between trials. Facilities routinely performing propulsion assessments may find it necessary to designate an assessment area in which the setup will remain intact.

\section{Validity}

Concurrent validity results were more variable than the reliability outcomes. While there were no statistically significant differences among systems for any of the joint angle outcomes, concurrent validity between values was not as favorable. When comparing joint angle data, concurrent validity was good for only elbow flexion between the Kinect and Dartfish. This is reasonable, considering the limited two-dimensional capabilities of Dartfish and the complex nature of the shoulder joint with greater degrees of freedom. Joint angle comparisons between the Kinect and Motion Analysis also revealed good concurrent validity for elbow flexion alone. While good concurrent validity was revealed for elbow flexion overall, validity did vary by participant. Participant 5 demonstrated only slight variation among systems (Figure 2), whereas participant 4 demonstrated greater variation (Figure 3). See Figure 4 for visual representation of the previously described mixed methods approach used to address participant 4's outlying data with decreased variation.

Poor concurrent validity across the other joints may be explained. Neck and trunk flexion may have been difficult to measure because they are the smallest angles of all joint angle outcomes. Considering the Kinect's accepted accuracy limitations, measurements of smaller angles are more difficult to obtain. Poor concurrent validity at the shoulder joint for both flexion and abduction again may be attributed to the complexity and greater degrees of freedom. Concurrent validity of wrist flexion may be poor because the Kinect hand marker was utilized to measure wrist movement due to the close proximity of the two markers and more consistent tracking of the hand marker. Additionally, consistent tracking of the hand marker is compromised upon contact with the wheelrim during propulsion because the Kinect has difficulty distinguishing between the upper limb and the wheelchair.

Concurrent validity between push loop data was similar to that of the joint angles for the Kinect as compared with Dartfish, as well as the Kinect as compared with Motion Analysis. Maximum height and hand-axle relationship may have been most consistent across all sys-

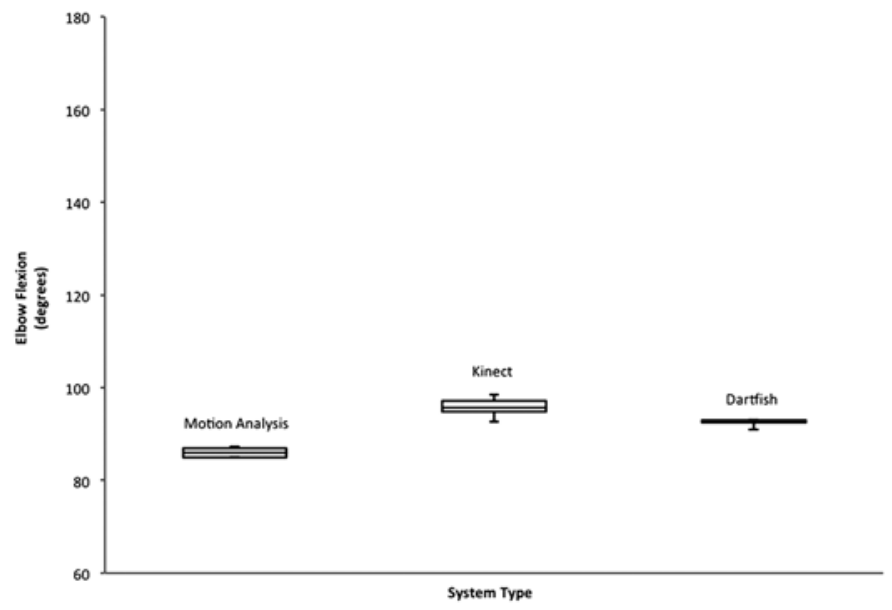

Figure 2.

Box and whisker plot of elbow flexion variation among systems for participant 5 in trial one of the first series of dynamic trials. tems since they are determined by the fixed points of the highest and lowest point on the push loop. Characteristics of maximum length and push length were determined by the more subjective manner in which the beginning and end of a push is defined. Time stamp variance also has the potential to contribute to poor concurrent validity between maximum length and push length. Dartfish in particular may have had difficulty calculating push length, considering it was an indirect outcome measure based on an initial calculation of push angle to be later converted to push length. Poor concurrent validity for push length may also be attributed to greater variation among systems across participants, as seen with elbow flexion and other variables. However, unlike with elbow flexion, participant 4 demonstrated only slight variation in push length (Figure 5), whereas participant 5 demonstrated greater variation (Figure 6). See Figure 7 for visual representation of the previously described mixed methods approach used to address participant 5 outlying data with decreased variation.

Maximum area is also an indirect measure, using the initial calculations of maximum length and maximum height for conversion by all systems. Good concurrent validity between the Kinect and Dartfish for maximum area measurement compared with the Kinect and Motion Analysis may then be explained by the more favorable pair of maximum length and maximum height ICC results for Dartfish and Kinect compared with Kinect and Motion Analysis. While both Dartfish and Kinect and 


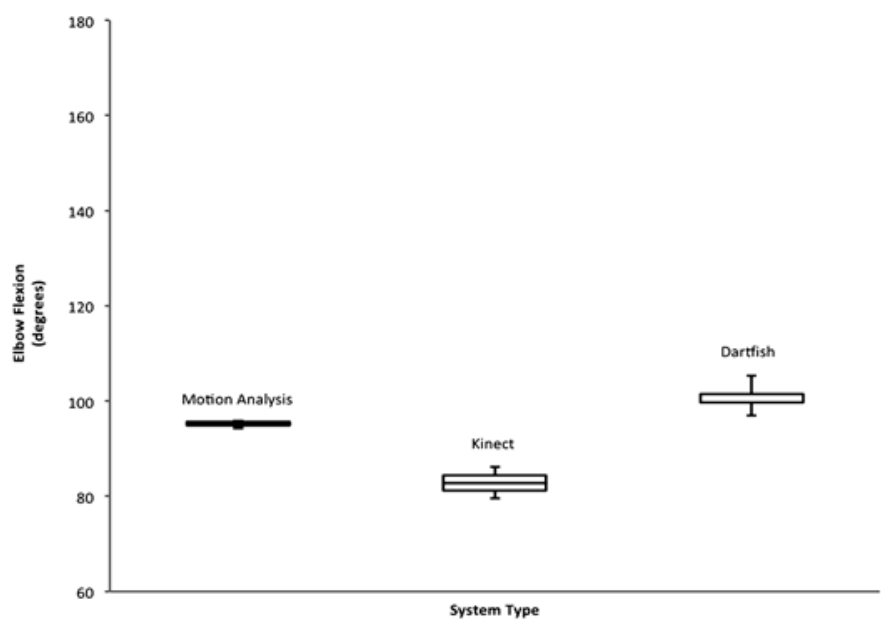

Figure 4.

Box and whisker plot of elbow flexion variation among systems for participant 4 following mixed methods approach to address outlying data in trial one of the first series of dynamic trials.

Kinect and Motion Analysis had good current validity for maximum height, better concurrent validity for maximum length between Kinect and Dartfish suggests that the two systems perhaps had more similar definitions of the beginning and end of a push compared with the Kinect and Motion Analysis.

Last, the lack of significant differences among systems for both joint angle and push loop data across dynamic trials speaks to a second clinical scenario represented by the power down in between Dynamic 1 and

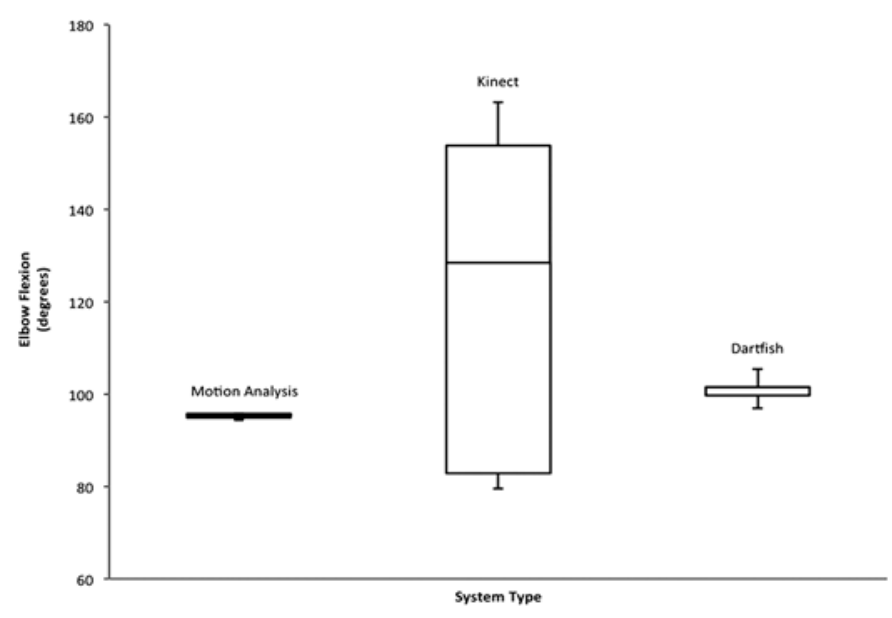

Figure 3.

Box and whisker plot of elbow flexion variation among systems for participant 4 in trial one of the first series of dynamic trials.

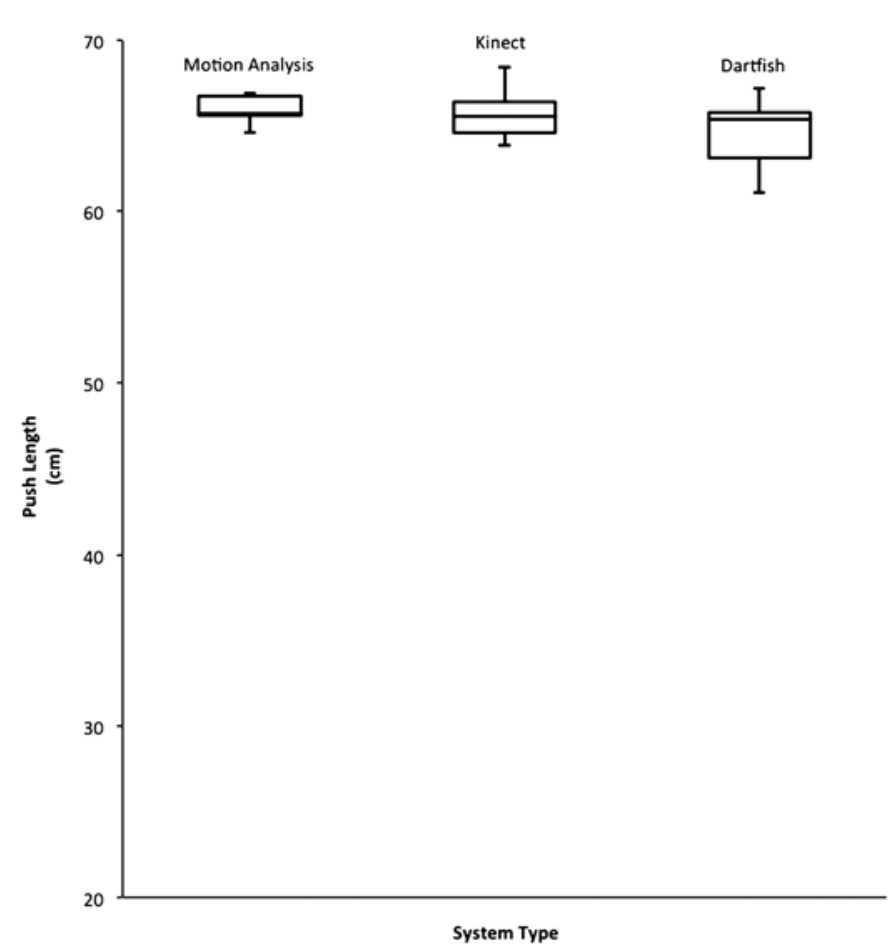

Figure 5.

Box and whisker plot of push length variation among systems for participant 4 in trial one of the first series of dynamic trials.

Dynamic 2 trials. Results suggest that differences between the Kinect, Dartfish, and Motion Analysis for dynamic 1 were not significantly different from the differences between the Kinect, Dartfish, and Motion Analysis for dynamic 2. Clinically, this supports the notion that facilities may designate an area for propulsion assessment and maintain the same setup from day to day with only a power up in between assessments without significantly affecting Kinect accuracy.

\section{Limitations}

This pilot study had limitations in its small sample size, the use of the systems themselves, and the methods of comparison. A limitation observed across all systems was the difference in frame rates at which data were collected. Different frame rates resulted in slightly different time stamps, affecting the ability to perfectly sync the beginning, middle, and end of each push. Also, the Kinect sensor is only able to capture a relatively small area in space. A small capture volume required the use of a roller system for the participant to remain stationary and within the space of the capture volume. Unfortunately, this does 


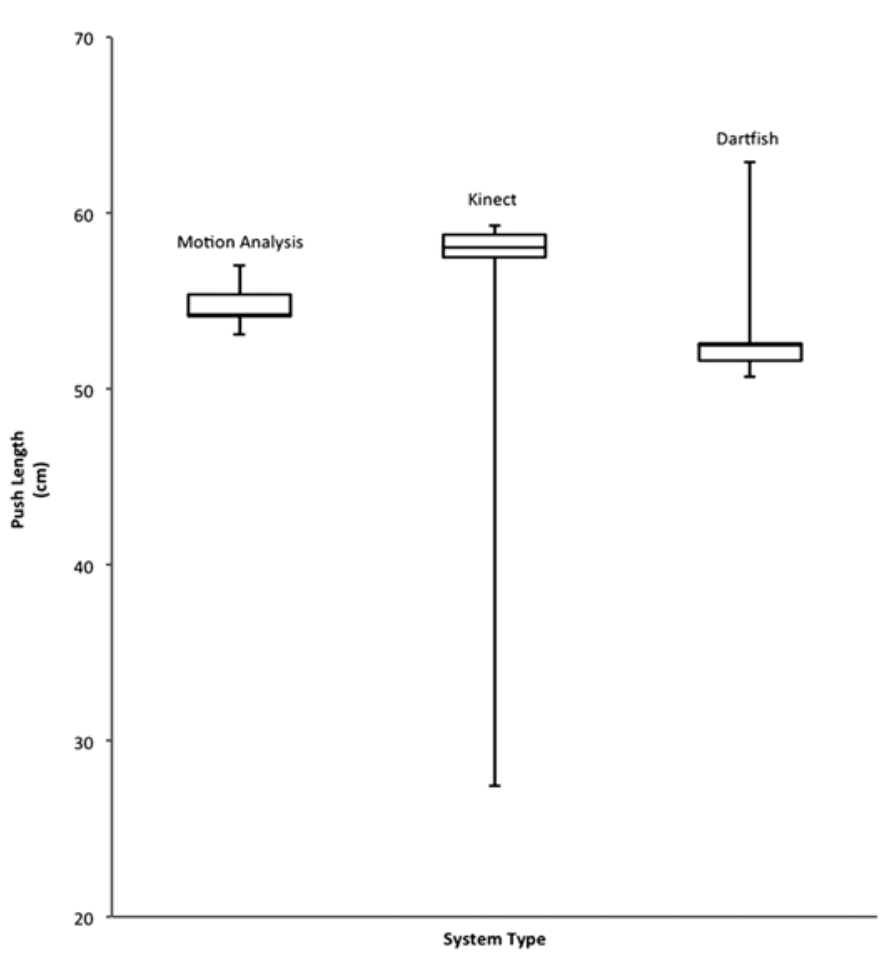

Figure 6.

Box and whisker plot of push length variation among systems for participant 5 in trial one of the first series of dynamic trials.

not allow the Kinect to effectively follow and assess a participant during more natural, and perhaps more clinically relevant, propulsion cycles performed overground. The Kinect is limited additionally in its true reflection of an individual's push pattern because the sensor and associated algorithms are unable to detect the presence of and differentiate a big loop and small loop in the event that a push pattern presents a crossover point, creating two loops; analysis is then limited to only the larger loop.

With regard to the wheelchair, the Kinect sensor was unable to register the physical components of the chair. Positioning of the wheel hub was attempted during Kinect calibration before data collection but was inconsistent among participants by several meters. Due to the lack of relationship established between participants and their wheelchairs, a series of algorithms must be developed to determine the wheelchair's position in space in comparison to the participant. Furthermore, the Kinect coordinate space differs from coordinate spaces for Dartfish and Motion Analysis. While the same variables are being calculated across systems, different coordinate spaces require different mathematical algorithms to obtain similar outcomes. Algo-

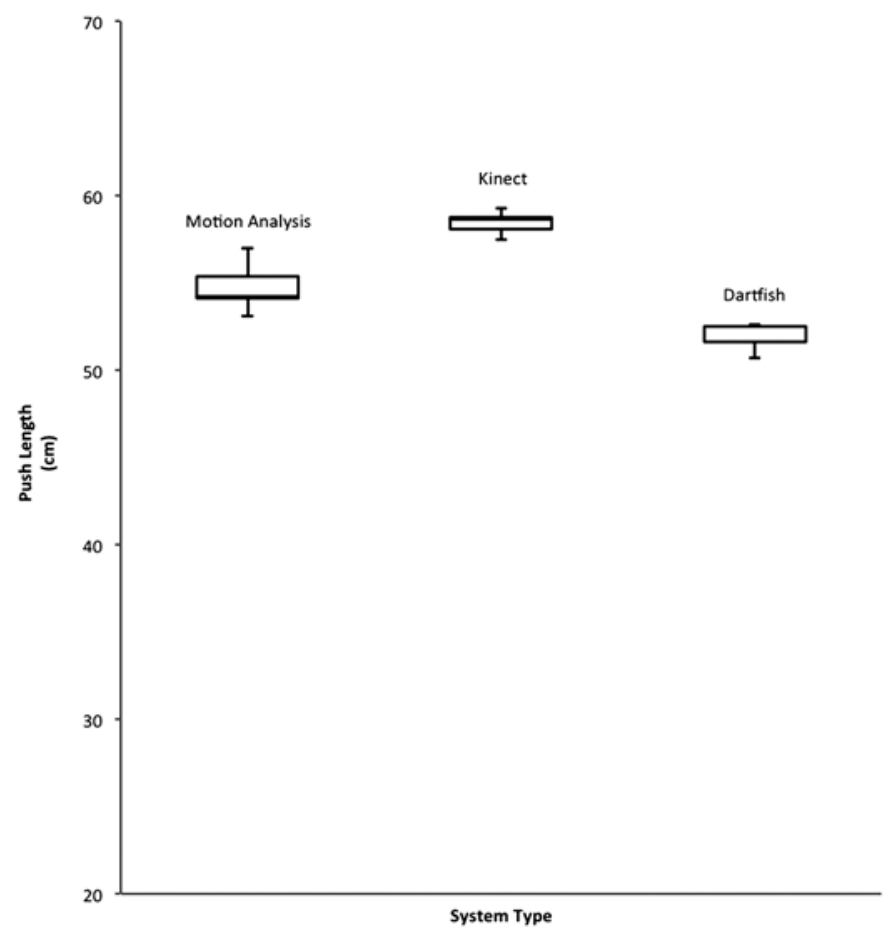

Figure 7.

Box and whisker plot of push length variation among systems for participant 5 following mixed methods approach for outlying data in trial one of the first series of dynamic trials.

rithm customization for the Kinect and Motion Analysis systems, along with manual Dartfish measurements, is time intensive and remains a limiting factor in the use of all three systems. However, even with customized algorithms, there is the potential that the Kinect may not apply equally to participants with a variety of diagnoses.

When an individual enters the Kinect sensor's field of view, a skeletal structure based on typical human proportions is applied to the individual, which serves as a frame of reference. Unfortunately, this skeletal structure may not be representative of all individuals in need of rehabilitation services. A participant in the current study was born with disproportionate limbs. As suggested by the flickering body segments of the skeletal structure during initial data collection, the sensor may not have been registering movement as accurately. A participant with varied proportions may suggest increased need for consideration of both left and right upper limbs. The current study was limited in its use of only one-sided data from the dominant side. Due to the angle at which the sensor was placed, the left upper limb was often 
obscured, potentially skewing shoulder midpoint data, thus affecting measurement of trunk flexion as well.

\section{Future Work}

Future work may aim to consider more efficient system and method utilization with a larger sample size. The Kinect Version 2 provides potential for increased accuracy and automation, with results of the current study serving as the platform. Combined use of the Kinect Version 2, the addition of LED lights to the pushrim of the wheelchair to increase visibility of the wheelchair [49-50], built-in tracking of a "trunk" vector, and sensors recording movement of both upper limbs may be worthwhile to investigate. Last, future studies utilizing reliable and valid methods may develop and package useful guidelines and customized algorithms for use in research and clinical practice among those with limited technical skills. Guidelines may include overall dimensions required for participants and all equipment, specified distance from sensor to floor and sensor to participant, specified sensor angle, and mixed methods approach to address outlying data for decreased variation.

This pilot study is consistent with previous literature comparing the Kinect and a three-dimensional goldstandard alternative in the assessment of postural control and gait [30-31,51]. Along with the present study, previous research has suggested varied accuracy across outcomes and unreliable body segment lengths affecting accuracy of joint positioning.

\section{CONCLUSIONS}

Preliminary evidence suggests that the Kinect is a sufficiently reliable assessment tool of manual wheelchair propulsion in terms of interrater and test-retest reliability. The Kinect has the potential to be a concurrently valid assessment tool, providing statistically comparable results to the human motion analysis gold standard with varying levels of agreement across outcome measures. Outcomes identified as insufficiently accurate compared with other systems may be addressed through further data processing and the development of consistent operational definitions with corresponding algorithms.

In combination with its cost-effective and portable features, the Kinect has the ability to promote the importance of propulsion assessment in MWUs by increasing the number of assessments performed, streamlining the process, and providing objective quantification in justifying rehabilitation services across a variety of settings.

\section{ACKNOWLEDGMENTS}

\section{Author Contributions:}

Study concept and design: R. Milgrom, J. R. Engsberg, K. A. Morgan. Acquisition of data: R. Milgrom, M. Foreman, J. Standeven, K. A. Morgan.

Analysis and interpretation of data: R. Milgrom, M. Foreman, J. Standeven, J. R. Engsberg, K. A. Morgan.

Drafting of manuscript: R. Milgrom, M. Foreman, J. Standeven, J. R. Engsberg, K. A. Morgan.

Critical revision of manuscript for important intellectual content: R. Milgrom, M. Foreman, J. Standeven, J. R. Engsberg, K. A. Morgan. Financial Disclosures: The authors have declared that no competing interests exist.

Funding/Support: This material was based on work supported by the Washington University Program in Occupational Therapy and Paraquad.

Additional Contributions: A special thank you to Kristin Will, Taniya Easow, Annie Donnelly, Sarah Adam, Holly Reitter, Rachel Tangen, Bill Janes, Christina Stephens, and Megen Devine for their assistance with this project. The authors would also like to thank the participants who took the time to participate in this project. Rachel Milgrom is now a registered and licensed occupational therapist. Institutional Review: The protocol for data collection and participant interaction was approved by the Washington University Human Research Protection Office. Informed written consent was obtained from all participants prior to participation in this study.

Participant Follow-Up: The authors do not plan to inform participants of the publication of this study.

\section{REFERENCES}

1. de los Reyes-Guzmán A, Dimbwadyo-Terrer I, TrincadoAlonso F, Monasterio-Huelin F, Torricelli D, Gil-Agudo A. Quantitative assessment based on kinematic measures of functional impairments during upper extremity movements: A review. Clin Biomech (Bristol, Avon). 2014;29(7):719-27. [PMID:25017296]

http://dx.doi.org/10.1016/j.clinbiomech.2014.06.013

2. Vito LD, Postolache O, Rapuano S. Measurements and sensors for motion tracking in motor rehabilitation. IEEE Instrum Meas Mag. 2014;17(3):30-38.

http://dx.doi.org/10.1109/MIM.2014.6825386

3. Yang Y, Pu F, Li Y, Li S, Fan Y, Li D. Reliability and validity of Kinect RGB-D sensor for assessing standing balance. IEEE Sens J. 2014;14(5):1633-38. http://dx.doi.org/10.1109/JSEN.2013.2296509

4. Aljure J, Eltorai I, Bradley WE, Lin JE, Johnson B. Carpal tunnel syndrome in paraplegic patients. Paraplegia. 1985; 
23(3):182-86. [PMID:4011294]

http://dx.doi.org/10.1038/sc.1985.31

5. Burnham RS, Steadward RD. Upper extremity peripheral nerve entrapments among wheelchair athletes: Prevalence, location, and risk factors. Arch Phys Med Rehabil. 1994; 75(5):519-24. [PMID:8185443]

6. Davidoff G, Werner R, Waring W. Compressive mononeuropathies of the upper extremity in chronic paraplegia. Paraplegia. 1991;29(1):17-24. [PMID:2023767] http://dx.doi.org/10.1038/sc.1991.3

7. Gellman H, Chandler DR, Petrasek J, Sie I, Adkins R, Waters RL. Carpal tunnel syndrome in paraplegic patients. J Bone Joint Surg Am. 1988;70(4):517-19. [PMID:3356717]

8. Wylie EJ, Chakera TM. Degenerative joint abnormalities in patients with paraplegia of duration greater than 20 years. Paraplegia. 1988;26(2):101-6. [PMID:3412779] http://dx.doi.org/10.1038/sc.1988.20

9. Akbar M, Balean G, Brunner M, Seyler TM, Bruckner T, Munzinger J, Grieser T, Gerner HJ, Loew M. Prevalence of rotator cuff tear in paraplegic patients compared with controls. J Bone Joint Surg Am. 2010;92(1):23-30.

[PMID:20048092]

http://dx.doi.org/10.2106/JBJS.H.01373

10. Dalyan M, Cardenas DD, Gerard B. Upper extremity pain after spinal cord injury. Spinal Cord. 1999;37(3):191-95. [PMID:10213328] http://dx.doi.org/10.1038/sj.sc.3100802

11. Collinger JL, Impink BG, Ozawa H, Boninger ML. Effect of an intense wheelchair propulsion task on quantitative ultrasound of shoulder tendons. PM R. 2010;2(10):920-25. [PMID:20970761]

http://dx.doi.org/10.1016/j.pmrj.2010.06.007

12. Rice I, Gagnon D, Gallagher J, Boninger M. Hand rim wheelchair propulsion training using biomechanical realtime visual feedback based on motor learning theory principles. J Spinal Cord Med. 2010;33(1):33-42.

[PMID:20397442]

http://dx.doi.org/10.1080/10790268.2010.11689672

13. Zhou H, Hu H. Human motion tracking for rehabilitationa survey. Biomed Signal Process Control. 2008;3(1):1-18. http://dx.doi.org/10.1016/j.bspc.2007.09.001

14. Boninger ML, Cooper RA, Baldwin MA, Shimada SD, Koontz A. Wheelchair pushrim kinetics: Body weight and median nerve function. Arch Phys Med Rehabil. 1999; 80(8):910-15. [PMID:10453767] http://dx.doi.org/10.1016/S0003-9993(99)90082-5

15. Boninger ML, Baldwin M, Cooper RA, Koontz A, Chan L. Manual wheelchair pushrim biomechanics and axle position. Arch Phys Med Rehabil. 2000;81(5):608-13.

[PMID:10807100]

http://dx.doi.org/10.1016/S0003-9993(00)90043-1
16. Boninger ML, Souza AL, Cooper RA, Fitzgerald SG, Koontz AM, Fay BT. Propulsion patterns and pushrim biomechanics in manual wheelchair propulsion. Arch Phys Med Rehabil. 2002;83(5):718-23. [PMID:11994814] http://dx.doi.org/10.1053/apmr.2002.32455

17. Rao SS, Bontrager EL, Gronley JK, Newsam CJ, Perry J. Three-dimensional kinematics of wheelchair propulsion. IEEE Trans Rehabil Eng. 1996;4(3):152-60.

[PMID:8800218] http://dx.doi.org/10.1109/86.536770

18. Newsam CJ, Rao SS, Mulroy SJ, Gronley JK, Bontrager EL, Perry J. Three dimensional upper extremity motion during manual wheelchair propulsion in men with different levels of spinal cord injury. Gait Posture. 1999;10(3):223-32.

[PMID:10567754] http://dx.doi.org/10.1016/S0966-6362(99)00034-X

19. Sturman DJ, Zeltzer D. A survey of glove-based input. IEEE Comput Graph Appl Mag. 1994;14(1):30-39. http://dx.doi.org/10.1109/38.250916

20. Eltoukhy M, Asfour S, Thompson C, Latta L. Evaluation of the performance of digital video analysis of human motion: Dartfish tracking system. Int J Sci Eng Res. 2012;3(3):1-6.

21. Bryson R. Virtual reality hardware. Implementing Virtual Reality, ACM SIGGRAPH. 1993;93:1-3.

22. Schmitz A, Ye M, Shapiro R, Yang R, Noehren B. Accuracy and repeatability of joint angles measured using a single camera markerless motion capture system. J Biomech. 2014;47(2):587-91. [PMID:24315287] http://dx.doi.org/10.1016/j.jbiomech.2013.11.031

23. Leow WK, Wang R, Leong HW. 3-D-2-D spatiotemporal registration for sports motion analysis. Mach Vis Appl. 2012;23(6):1177-94. http://dx.doi.org/10.1007/s00138-011-0371-7

24. Vanlandewijck YC, Verellen J, Tweedy S. Towards evidence-based classification in wheelchair sports: Impact of seating position on wheelchair acceleration. J Sports Sci. 2011;29(10):1089-96. [PMID:21756128] http://dx.doi.org/10.1080/02640414.2011.576694

25. Hayrinen M, Juntunen J, Blomqvist M, Overmark S, Molik B, Kosmol A, Morgulec-Adamowicz N. Match analysis of elite ice sledge hockey in Paralympics 2010. J Quan Anal Sport. 2011;7(3).

26. Lauterbach SA, Foreman MH, Engsberg JR. Computer games as therapy for persons with stroke. Games Health J. 2013;2(1):24-28. [PMID:26196551] http://dx.doi.org/10.1089/g4h.2012.0032

27. Chang YJ, Han WY, Tsai YC. A Kinect-based upper limb rehabilitation system to assist people with cerebral palsy. Res Dev Disabil. 2013;34(11):3654-59. [PMID:24012594] http://dx.doi.org/10.1016/j.ridd.2013.08.021

28. González-Ortega D, Díaz-Pernas FJ, Martínez-Zarzuela M, Antón-Rodríguez M. A Kinect-based system for cognitive 
rehabilitation exercises monitoring. Comput Methods Programs Biomed. 2014;113(2):620-31. [PMID:24263055]

http://dx.doi.org/10.1016/j.cmpb.2013.10.014

29. Hsieh WM, Chen CC, Wang SC, Tan SY, Hwang YS, Chen SC, Lai JS, Chen YL. Virtual reality system based on Kinect for the elderly in fall prevention. Technol Health Care. 2014;22(1):27-36. [PMID:24361986]

30. Clark RA, Pua YH, Fortin K, Ritchie C, Webster KE, Denehy L, Bryant AL. Validity of the Microsoft Kinect for assessment of postural control. Gait Posture. 2012;36(3): 372-77. [PMID:22633015] http://dx.doi.org/10.1016/j.gaitpost.2012.03.033

31. Clark RA, Bower KJ, Mentiplay BF, Paterson K, Pua YH. Concurrent validity of the Microsoft Kinect for assessment of spatiotemporal gait variables. J Biomech. 2013;46(15): 2722-25. [PMID:24016679] http://dx.doi.org/10.1016/j.jbiomech.2013.08.011

32. Mentiplay BF, Clark RA, Mullins A, Bryant AL, Bartold S, Paterson K. Reliability and validity of the Microsoft Kinect for evaluating static foot posture. J Foot Ankle Res. 2013; 6(1):14. [PMID:23566934] http://dx.doi.org/10.1186/1757-1146-6-14

33. Yeung LF, Cheng KC, Fong CH, Lee WC, Tong KY. Evaluation of the Microsoft Kinect as a clinical assessment tool of body sway. Gait Posture. 2014;40(4):532-38.

[PMID:25047828]

http://dx.doi.org/10.1016/j.gaitpost.2014.06.012

34. Chang CY, Lange B, Zhang M, Koenig S, Requejo P, Somboon N, Sawchuk A, Rizzo A. Towards pervasive physical rehabilitation using Microsoft Kinect. Proceedings of the 6th International Conference on Pervasive Computing Technologies for Healthcare (PervasiveHealth); 2012 May 21-24; San Diego, CA.

35. Okada Y, Shibata T, Tamei T, Orito Y, Funaya H, Obayashi C, Ikeda K, Hayamizu M, Morioka S. In-home posture evaluation and visual feedback training to improve posture with a Kinect-based system in Parkinson's disease: A case study. J Nov Physiother. 2014;4(5). http://dx.doi.org/10.4172/2165-7025.1000232

36. Metcalf CD, Robinson R, Malpass AJ, Bogle TP, Dell TA, Harris C, Demain SH. Markerless motion capture and measurement of hand kinematics: Validation and application to home-based upper limb rehabilitation. IEEE Trans Biomed Eng. 2013;60(8):2184-92. [PMID:23475333] http://dx.doi.org/10.1109/TBME.2013.2250286

37. Su CJ, Chiang CY, Huang JY. Kinect-enabled home-based rehabilitation system using Dynamic Time Warping and fuzzy logic. Appl Soft Comput. 2014;22:652-66. http://dx.doi.org/10.1016/j.asoc.2014.04.020

38. Gregory CM, Embry A, Perry L, Bowden MG. Quantifying human movement across the continuum of care: From lab to clinic to community. J Neurosci Methods. 2014;231:18-21.

\section{[PMID:24797226]}

http://dx.doi.org/10.1016/j.jneumeth.2014.04.029

39. Mobini A, Behzadipour S, Saadat Foumani M. Accuracy of Kinect's skeleton tracking for upper body rehabilitation applications. Disabil Rehabil Assist Technol. 2014;9(4): 344-52. [PMID:23786360] http://dx.doi.org/10.3109/17483107.2013.805825

40. Wu G, van der Helm FC, Veeger HE, Makhsous M, Van Roy P, Anglin C, Nagels J, Karduna AR, McQuade K, Wang X, Werner FW, Buchholz B; International Society of Biomechanics. ISB recommendation on definitions of joint coordinate systems of various joints for the reporting of human joint motion-Part II: Shoulder, elbow, wrist and hand. J Biomech. 2005;38(5):981-92. [PMID:15844264] http://dx.doi.org/10.1016/j.jbiomech.2004.05.042

41. Winter DA. Biomechanics and motor control of human movement. 4th ed. Hoboken (NJ): John Wiley and Sons, 2009.

42. Stephens CL, Engsberg JR. Comparison of overground and treadmill propulsion patterns of manual wheelchair users with tetraplegia. Disabil Rehabil Assist Technol. 2010; 5(6):420-27. [PMID:20441443] http://dx.doi.org/10.3109/17483101003793420

43. Will K, Engsberg JR, Foreman M, Klaesner J, Birkenmeier $\mathrm{R}$, Morgan K. Repetition-based training for efficient propulsion in new manual wheelchair users. Am J Occupation Ther;69(1):1.

44. Morgan KA, Engsberg JR, Gray DB. Important wheelchair skills for new manual wheelchair users: Health care professional and wheelchair user perspectives. Disabil Rehabil Assist Technol. 2015;2015:1-11. [PMID:26138222] http://dx.doi.org/10.3109/17483107.2015.1063015

45. Klaesner J, Morgan KA, Gray DB. The development of an instrumented wheelchair propulsion testing and training device. Assist Technol. 2014;26(1):24-32.

[PMID:24800451]

http://dx.doi.org/10.1080/10400435.2013.792020

46. ElAli TS. Discrete systems and digital signal processing with MATLAB. 2nd ed. Boca Raton (FL): CRC Press; 2012.

47. Portney LG, Watkins MP. Foundations of clinical research: applications to practice. Saddle River (NJ): Prentice Hall; 2009.

48. Rodgers MM, Gayle GW, Figoni SF, Kobayashi M, Lieh J, Glaser RM. Biomechanics of wheelchair propulsion during fatigue. Arch Phys Med Rehabil. 1994;75(1):85-93. [PMID:8291970]

49. Zhang S, He W, Yu Q, Zheng X. Low-cost interactive whiteboard using the Kinect. 2012 International Conference on Image Analysis and Signal Processing (IASP); 2012 Nov 9-11; Hangzhou, China. IEEE; 2012. p. 1-5. 
50. Hadjakos A. Pianist motion capture with the Kinect depth camera. Proceedings of the International Conference on Sound and Music Computing; 2012 Jan; Copenhagen, Denmark.

51. Weber I, Koch J, Meskemper J, Friedl K, Heinrich K, Hartmann U. Is the MS Kinect suitable for motion analysis? Biomed Tech (Berl). 2012;2012:57.

Submitted for publication October 19, 2015. Accepted in revised form March 29, 2016.
This article and any supplementary material should be cited as follows:

Milgrom R, Foreman M, Standeven J, Engsberg JR, Morgan KA. Reliability and validity of the Microsoft Kinect for assessment of manual wheelchair propulsion. J Rehabil Res Dev. 2016;53(6):901-18.

http://dx.doi.org/10.1682/JRRD.2015.10.0198

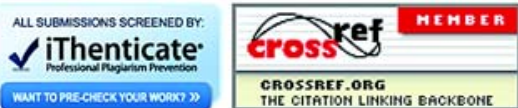


